Proc. 15th Int. Conference on Defects Recognition, Imaging and Physics in Semiconductors, Warsaw, Poland 2013

\title{
Defect Analysis of Pentacene Diode
}

\author{
L. Stuchlíková*, M. Weis*, P. Juhász, A. Kósa, L. Harmatha and J. JakaboviC \\ Slovak University of Technology in Bratislava, Faculty of Electrical Engineering and Information Technology \\ Institute of Electronics and Photonics, Ilkovičova 3, 81219 Bratislava, Slovak Republic
}

This paper demonstrates the analysis of defect states in pentacene film sandwiched between $\mathrm{Au}$ and $\mathrm{Al}$ electrodes by the deep-level transient spectroscopy method. Three hole-like deep energy levels were observed. The effective mass obtained from the simulation is applied and defect parameters, namely the capture cross-sections and the activation energy $3.7 \times 10^{-18} \mathrm{~cm}^{2}$ at $0.34 \mathrm{eV}, 3.1 \times 10^{-17} \mathrm{~cm}^{2}$ at $0.41 \mathrm{eV}$, and $2.9 \times 10^{-15} \mathrm{~cm}^{2}$ at $0.63 \mathrm{eV}$ is determined from the Arrhenius plot. Reliability of obtained defect parameters is confirmed by simulation of deep level transient spectra and comparison with experiment.

DOI: $10.12693 /$ APhysPolA.125.1038

PACS: 72.80.Le, 73.50.Gr, 71.55.-i

\section{Introduction}

Semiconductor devices based on organic materials, such as organic field effect transistors (OFETs), organic light-emitting diodes (OLEDs), and organic solar cells, have recently attracted research interests [1-4]. Recent research on organic devices has been concentrated on the applied research and focused on performance improvement. However, the fundamental research such as the charge transport mechanism investigation is also important. Although there are some similarities to the inorganic semiconductor devices, the principles of operation of devices based on organic semiconductors are different $[5,6]$. In other words, since the organic electronic devices have no doping, all mobile charges are injected from electrodes and transported through organic film [7]. As a result, the charge injection, carriers transport, and carriers trapping play a key role for device application.

Furthermore, the charge carriers trapping has been reported as one of the limitation factors of effective charge mobility [8]. The charge trap density in organic semiconductors reaches the value of the order of $10^{18} / \mathrm{cm}^{3}$ due to a high sensitivity of these materials to the ambient environment, as well as an imperfect material crystallinity. In other words, the organic materials exhibit presence of chemical defects as well as structural defects. Hence, further improvement of device performance depends on detailed understanding of present limitations.

The localized levels created between highest occupied molecular orbital (HOMO) and lowest unoccupied molecular orbital (LUMO) are assigned to the charge trapping phenomenon. For defects that are electrically active, techniques such as thermally stimulated current, impedance spectroscopy, or deep level transient spectroscopy (DLTS) have been successfully used for determining the trap parameters in inorganic semiconductors [9]. However, the application of these techniques

*corresponding author; e-mail: lubica.stuchlikova@stuba.sk, martin.weis@stuba.sk for organic semiconductors and following data analysis are still not fully described.

The DLTS method investigates the relaxation processes of charge carrier capture and emission from localized states (deep energy levels) [9]. It has been used for determination of the fundamental parameters of deep energy levels - activation energy $E_{\mathrm{T}}$ and capture cross-section $\sigma_{\mathrm{T}}$. Even though there are many experimental [10-13] and theoretical [14-16] reports on activation energies of defects, the capture cross-sections of charge carriers have not been determined yet. Therefore, the detailed characterisation of defects requires further research.

The aim of this work is to demonstrate the results obtained by the DLTS experiments carried out on pentacene diode structure. The hole effective mass obtained from the molecular modelling is applied to evaluate the capture cross-sections and make subsequent discussion on defect properties. The simulation of the DLTS signal using the obtained defect parameters has been also performed.

\section{Experimental details and methods}

The devices used in this study were $\mathrm{Al} /$ pentacene/ $\mathrm{Au}$ sandwich structures as depicted in Fig. 1a. The glass plates made of the Corning 1737 glass with a near zero alkali-alumino-silicate content have been used as substrates. Prior the thin film deposition, the substrates were washed in ultrasonic bath by isopropanol and pure water, consecutively, and then cleaned up by oxygen plasma. All the layers have been prepared by the thermal evaporation in a vacuum better than $8 \times 10^{-6}$ Torr through the shadow masks to determine the device area $\left(0.49 \mathrm{~mm}^{2}\right)$. Pentacene (> 98\%, Acros Organics) layer $260 \mathrm{~nm}$ thick has been deposited at a constant evaporation rate of $0.5 \mathrm{~nm} / \mathrm{min}$. The $\mathrm{Al}$ and $\mathrm{Au}$ layers had thicknesses of 80 and $65 \mathrm{~nm}$, respectively.

Electrically active defects and emission processes have been studied by the DLTS technique using the Fourier analysis of the measured capacitance transients (deep level transient Fourier spectroscopy, DLTFS). The Fourier transformation and the evaluation of the time 


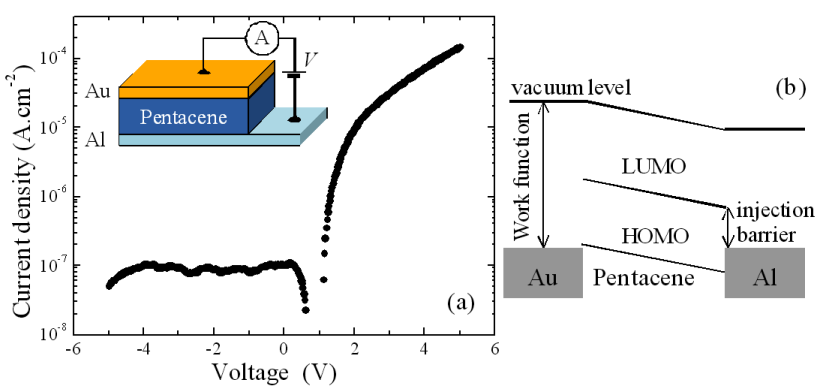

Fig. 1. (a) The current density-voltage dependence of $\mathrm{Au} /$ pentacene/ $\mathrm{Al}$ device at temperature of $300 \mathrm{~K}$. Inset illustrates fabricated structure. (b) Simplified view of the energy band structure at zero bias.

constant as well as the transient amplitude have been applied for every transient measured at given temperature. From the analysis of the transients measured during a temperature scan the Fourier coefficients $a_{n}$ and $b_{n}$ of $n$-th order are determined and plotted as the DLTS spectrum [17]. In contrast to the rate window approach, the Fourier analysis based DLTS is more sensitive and precise $[17,18]$. These electrical measurements were carried out using a BIORAD DL8000 measuring system. Thirty six sets of DLTFS spectra were measured. In DLTFS experiments wide ranges of reverse voltage biases (from -1 to $2.5 \mathrm{~V}$ ), pulse voltages (from 1 to $3 \mathrm{~V}$ ), temperatures (from 90 to $300 \mathrm{~K}$ ), and times of the filling bias pulses (from 0.5 to $3 \mathrm{~ms}$ ) were employed to investigate charge relaxation.

\section{Results and discussion}

The energy band diagram illustrating $\mathrm{Au} /$ pentacene/Al device is depicted in Fig. 1b. The difference between the metal work function and the HOMO (LUMO) energy level represents the injection barrier for holes (electrons). Since HOMO and LUMO energies for pentacene are 5.0 and $3.2 \mathrm{eV}$ [19], respectively, the metals $\mathrm{Au}(5.3 \mathrm{eV})$ and $\mathrm{Al}(4.2 \mathrm{eV})$ [20] have been chosen as the electrode materials for low and high level of the charge carriers injection into pentacene, respectively (Fig. 1b).

Figure 1 shows the current density versus voltage measurements of the device at the temperature of $300 \mathrm{~K}$ and confirms rectifying behaviour of the fabricated structure with rectification ratio of $10^{3}$ in the applied voltage region. Subsequently, the capacitance transients have been recorded at temperatures ranging from 90 to $300 \mathrm{~K}$. Typical DLTS spectra are demonstrated in Fig. 2 for the following setup: time period $T_{\mathrm{w}}=0.6 \mathrm{~s}$, filling time $t_{\mathrm{P}}=1 \mathrm{~s}$, filling pulse voltage $V_{\mathrm{P}}=3 \mathrm{~V}$, and reverse voltage $V_{\mathrm{R}}=-0.05 \mathrm{~V}$.

Obviously, the obtained DLTS spectra represent the sum of multiple relaxations, related to emission processes from deep energy levels. The emission processes from traps denoted as T1, T2, and T3 are most significant at

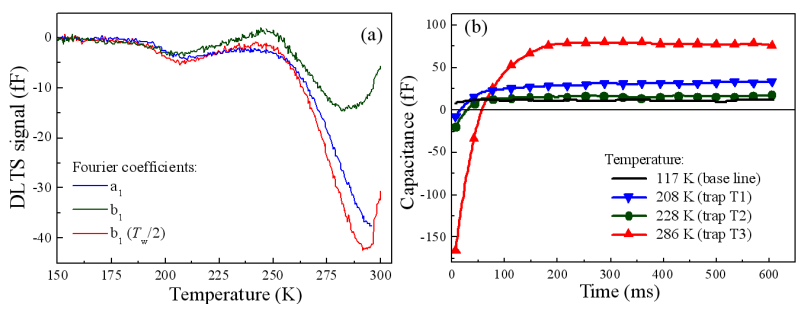

Fig. 2. (a) DLTS spectra for Fourier coefficients $a_{1}$, $b_{1}$, and $b_{1}\left(T_{\mathrm{w}} / 2\right)$ measured for time period $T_{\mathrm{w}}=0.6 \mathrm{~s}$, filling time $t_{\mathrm{P}}=1 \mathrm{~s}$, filling pulse voltage $V_{\mathrm{P}}=3 \mathrm{~V}$, and reverse voltage $V_{\mathrm{R}}=-0.05 \mathrm{~V}$. (b) Recorded capacitance transients for temperatures at peak positions $(208,228$, and $286 \mathrm{~K}$ ) and at $117 \mathrm{~K}$.

temperatures of 208, 228, and $286 \mathrm{~K}$, respectively. Figure $2 \mathrm{~b}$ depicts capacitance transients for these temperatures as well as at the temperature of $117 \mathrm{~K}$ which can be used as a base line (no peak was detected in the DLTS spectra at this temperature). All transients recorded at the temperatures corresponding to peak positions exhibit an exponential behaviour (regression coefficient of 0.97 , 0.96 and 0.99 ) in contrast to the transient measured at $117 \mathrm{~K}$, which does not show exponential decay. The Arrhenius plots shown in Fig. 3 reveal their activation energies of $E_{\mathrm{T} 1}=0.34 \mathrm{eV}, E_{\mathrm{T} 2}=0.41 \mathrm{eV}$, and $E_{\mathrm{T} 3}=0.63 \mathrm{eV}$.

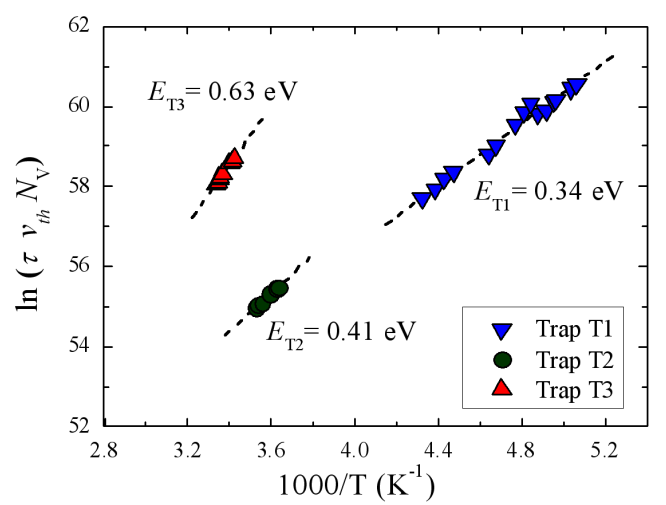

Fig. 3. Arrhenius plots for deep levels T1, T2, and T3.

Moreover, the Arrhenius plot can provide the capture cross-section $(\sigma)$ using the relation

$$
\ln \left(\frac{e_{i}}{T^{2}}\right)=\ln \left(\sigma_{i} K_{i}\right)-\left(\frac{\Delta E_{\mathrm{T}}}{k_{\mathrm{B}} T}\right),
$$

where $e_{i}, K_{i}, T$, and $k_{\mathrm{B}}$ are the emission rate of $i$-th trap, material coefficient corresponding to $i$-th trap, temperature and the Boltzmann constant. The material coefficient $K_{i}$ is dependent on the effective mass $m^{*}$ as follows:

$$
K_{i}=3^{1 / 2} 2^{5 / 2} \pi^{3 / 2} \frac{k_{\mathrm{B}}^{2} m_{\mathrm{s}}^{* 3 / 2}}{h^{3} m^{* 1 / 2}},
$$

where $m_{\mathrm{s}}^{*}$ is the density-of-states effective mass. Because there is no experimental estimation of the effective mass, the reported molecular modelling provided the effective mass values of $1.30 m_{0}$ [21], 3.31 $m_{0}$ [22], and $5.52 m_{0}$ [23]. 
Capture cross-section evaluated using these values are summarized in Table I.

The capture cross-sections for three values

TABLE I of hole effective masses.

\begin{tabular}{c|c|c|c|c}
\hline \hline \multirow{2}{*}{$\begin{array}{c}\text { Deep } \\
\text { level }\end{array}$} & $\begin{array}{c}\text { Activation } \\
\text { energy } E_{\mathrm{T}}[\mathrm{eV}]\end{array}$ & \multicolumn{3}{|c}{ Capture cross-section $\sigma_{\mathrm{T}}\left[\mathrm{cm}^{2}\right]$} \\
\cline { 3 - 5 } & $m^{*} / m_{0}=1.3$ & $m^{*} / m_{0}=3.31$ & $m^{*} / m_{0}=5.52$ \\
\hline T1 & 0.34 & $8.32 \times 10^{-18}$ & $3.7 \times 10^{-18}$ & $2.08 \times 10^{-18}$ \\
T2 & 0.41 & $3.56 \times 10^{-18}$ & $3.1 \times 10^{-17}$ & $1.09 \times 10^{-17}$ \\
T3 & 0.63 & $5.16 \times 10^{-15}$ & $2.9 \times 10^{-15}$ & $1.29 \times 10^{-15}$
\end{tabular}

Noticeably, although the higher value of effective mass leads to lower effective capture cross-section, the differences are within one order of magnitude and they demonstrate the error of the estimation. It should be added here that the thermal velocity, $v_{\text {th }}=\left(3 k_{\mathrm{B}} T / m^{*}\right)^{1 / 2}$, varies in the range from $5 \times 10^{6}$ to $1 \times 10^{7} \mathrm{~cm} / \mathrm{s}$, which, to some extent may influence the analysis. Table II presents the deep level parameters calculated for the assumed value of the effective mass $m^{*} / m_{0}=3.31$. These results were verified by evaluation of more than ten sets of measured DLTS spectra.

Parameters of the deep levels observed

TABLE II in $\mathrm{Au} /$ pentacene/Al structure.

\begin{tabular}{|c|c|c|c|}
\hline $\begin{array}{l}\text { Deep } \\
\text { level }\end{array}$ & $\begin{array}{c}\text { Activation } \\
\text { energy } \\
E_{\mathrm{T}}[\mathrm{eV}]\end{array}$ & $\begin{array}{l}\text { Cross-section } \\
\sigma_{\mathrm{T}}\left[\mathrm{cm}^{2}\right]\end{array}$ & Reference \\
\hline $\mathrm{T} 1$ & 0.34 & $3.68 \times 10^{-18}$ & $\begin{array}{c}\text { hydrogen-related defect } \\
0.34 \mathrm{eV} \text { by DFT [14] }\end{array}$ \\
\hline $\mathrm{T} 2$ & 0.41 & $3.12 \times 10^{-17}$ & $\begin{array}{c}\text { water-related defect } \\
0.43 \mathrm{eV} \text { by VRH/TRM }[15,16]\end{array}$ \\
\hline T3 & 0.63 & $2.92 \times 10^{-15}$ & $\begin{array}{l}\text { oxygen-related defect } \\
0.62 \mathrm{eV} \text { by DFT [14] }\end{array}$ \\
\hline
\end{tabular}

The deep energy level T1 $\left(E_{\mathrm{T} 1}=0.34 \mathrm{eV}\right)$ is in good agreement with the reported trap activation energy of $0.34 \mathrm{eV}$, which has been assigned to the hydrogen defect (dihydropentacene, $\mathrm{C}-\mathrm{H}_{2}$ ) based on density functional theory (DFT) calculations [14]. The deep energy level $\mathrm{T} 2\left(E_{\mathrm{T} 2}=0.41 \mathrm{eV}\right)$ corresponds to water-related defect with the activation energy of $0.43 \mathrm{eV}$ obtained from variable range hopping between localized states and multiple trapping and release model (VRH-TRM) [15, 16]. The third deep energy level T3 $\left(E_{\mathrm{T} 3}=0.63 \mathrm{eV}\right)$ has been reported as the defect related to the oxygen atom (pentacenequinone, $\mathrm{C}=\mathrm{O}$ ) with the activation energy equal to $0.62 \mathrm{eV}$. This value was determined by DFT calculations [14]. The evaluated deep level parameters have also been used for simulations of the DLTS spectra.

Figure 4 presents the simulated DLTS spectra for the Fourier coefficient $b_{1}\left(T_{\mathrm{w}} / 2\right)$ corresponding to the above-mentioned measurement conditions. The good agreement between the simulation results and experimental data confirms reliability of the estimated defect parameters.

\section{Conclusion}

In this work the thermal emission processes in the $\mathrm{Au} /$ pentacene/Al sandwich structure have been investi-

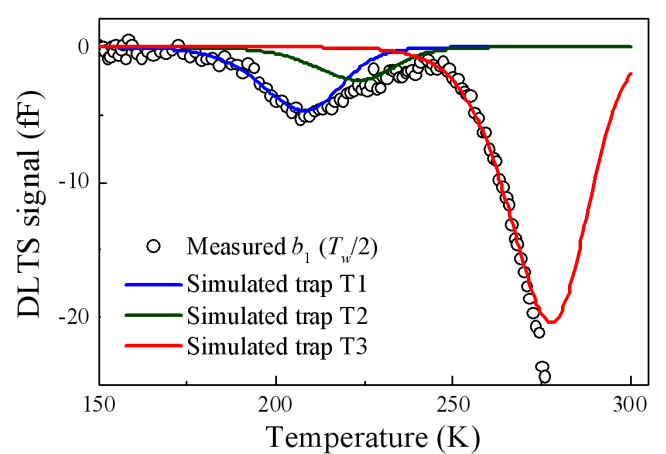

Fig. 4. Simulated (solid lines) and measured (open circles) DLTS spectra for the Fourier coefficient $b_{1}\left(T_{\mathrm{w}} / 2\right)$ corresponding to the following measurement conditions: time period $T_{\mathrm{w}}=0.6 \mathrm{~s}$, filling time $t_{\mathrm{P}}=1 \mathrm{~s}$, filling pulse voltage $V_{\mathrm{P}}=3 \mathrm{~V}$, and reverse voltage $V_{\mathrm{R}}=-0.05 \mathrm{~V}$. The simulations are based on the parameters of the identified deep levels labeled as T1, T2, and T3.

gated by the DLTS method, based on the Fourier transform analysis of the measured capacitance transients under various experimental conditions. Three hole-like deep energy levels T1, T2, and T3 have been found. The deep level $\mathrm{T} 1$ with the activation energy $E_{\mathrm{T} 1}=$ $0.34 \mathrm{eV}$ and capture cross-section $\sigma_{1}=3.7 \times 10^{-18} \mathrm{~cm}^{2}$ has been assigned to the hydrogen-related defect (dihydropentacene). The deep level T2 with the activation energy $E_{\mathrm{T} 2}=0.41 \mathrm{eV}$ and capture cross-section $\sigma_{2}=3.1 \times 10^{-17} \mathrm{~cm}^{2}$ corresponds to a water-related trap state. The third level T3 with the activation energy $E_{\mathrm{T} 3}=0.63 \mathrm{eV}$ and capture cross-section $\sigma_{3}=$ $2.9 \times 10^{-15} \mathrm{~cm}^{2}$, has been assigned to the oxygen-related defect (pentacenequinone). The estimated defect parameters have been verified by the simulation of the DLTS spectra and their comparison with the experimental spectra. These results demonstrate the application of the capacitance DLTS technique with the Fourier analysis, as a method, suitable for the studies of defects in organic semiconductors and illustrate its potentialities for verification of theoretical models.

\section{Acknowledgments}

The work has been supported by the Scientific Grant Agency of the Ministry of Education, Science, Research and Sport of the Slovak Republic and of the Slovak Academy of Sciences (projects VEGA 1/0866/11, $1 / 0377 / 13$ and VEGA $1 / 0851 / 11$ ) and the Slovak Research and Development Agency (APVV-0262-10).

\section{References}

[1] H.E. Katz, J. Mater. Chem. 7, 369 (1997).

[2] H.E. Katz, Chem. Mater. 16, 4748 (2004).

[3] H. Sirringhaus, Adv. Mater. 17, 2411 (2005).

[4] M. Mas-Torrent, C. Rovira, Chem. Soc. Rev. 37, 827 (2008). 
[5] N. Karl, Synth. Met. 133-134, 649 (2003).

[6] V. Coropceanu, J. Cornil, D.A. de Silva, Y. Olivier, R. Silbey, J.-L. Brédas, Chem. Rev. 107, 926 (2007).

[7] G. Horowitz, in: Organic Electronics, Ed. H. Klauk, Wiley-VCH, Weinheim 2006.

[8] T. Manaka, F. Liu, M. Weis, M. Iwamoto, J. Appl. Phys. 107, 043712 (2010).

[9] D.V. Lang, J. Appl. Phys. 45, 3023 (1974).

[10] A.J. Campbell, D.D.C. Bradley, E. Werner, W. Brütting, Synth. Met. 111-112, 273 (2000).

[11] Y.S. Yang, S.H. Kim, J.-I. Lee, H.Y. Chu, L.-M. Do, H. Lee, J. Oh, T. Zyung, Appl. Phys. Lett. 80, 1595 (2002).

[12] S. Neugebauer, J. Rauh, C. Deibel, V. Dyakonov, Appl. Phys. Lett. 100, 263304 (2012).

[13] T.P. Nguyen, C. Renaud, P. Le Rendu, S.H. Yang, Phys. Status Solidi C 6, 1856 (2009).

[14] J.E. Northrup, M.L. Chabinyc, Phys. Rev. B 68, 041202 (2003).
[15] K.P. Pernstich, D. Oberhoff, C. Goldmann, B. Batlogg, Appl. Phys. Lett. 89, 213509 (2006).

[16] D. Oberhoff, K.P. Pernstich, D.J. Gundalch, B. Batlogg, IEEE Trans. Electron. Dev. 54, 17 (2007).

[17] S. Weiss, R. Kassing, Solid-State Electron. 31, 1733 (1988).

[18] R. Bayer, H. Burghardt, E. Thomas, R. Reich, D.R.T. Zahn, T. Gessner, Microelectron. Reliab. 39, 297 (1999).

[19] T. Yasuda, T. Goto, K. Fujita, T. Tsutsui, Appl. Phys. Lett. 85, 2098 (2004).

[20] D.R. Lide, CRC Handbook of Chemistry and Physics, 84th ed., CRC Press, 2003.

[21] K. Hummer, C. Ambrosh-Draxl, Phys. Rev. B 72, 205205 (2005).

[22] H. Yoshida, N. Sato, Phys. Rev. B 77, 235205 (2008).

[23] G.A. de Wijs, C.C. Mattheus, R.A. de Groot, T.T.M. Palstra, Synth. Met. 139, 109 (2003). 